\title{
Estimating Glomerular Filtration Rate in African American Individuals
}

Pierre Delanaye ${ }^{a, b}, \mathrm{MD}, \mathrm{PhD}$; Hans Pottel ${ }^{\mathrm{c}}, \mathrm{PhD}$

a CHU Sart Tilman, Department of Nephrology-Dialysis-Transplantation, University of Liège, Liège, Belgium

${ }^{\mathrm{b}}$ Department of Nephrology-Dialysis-Apheresis, Hopital Universitaire Caremeau, Nimes, France

c Department of Public Health and Primary Care, KU Leuven Campus Kulak Kortrijk, Kortrijk, Belgium Corresponding Author: Pierre Delanaye, MD, PhD, Service de Dialyse, CHU Sart Tilman, Department of Nephrology-Dialysis-Transplantation, University of Liège, 4000 Liège, Belgium (pierre_delanaye@yahoo.fr) 


\section{To the Editor}

Levey et $\mathrm{al}^{1}$ argue that the Chronic Kidney Disease Epidemiology Collaboration (CKD-EPI) equation is the most accurate tool for determining glomerular filtration rate (GFR) in African American people. However, this conclusion is questionable. First, as we established in 2011, the current CKD-EPI equations with the ethnic African American coefficient underestimate GFR in African American patients with chronic kidney disease (CKD) but overestimate it in African American patients who do not have CKD. ${ }^{2}$ Because the mean bias of this equation crosses the line of zero bias, it is quite challenging to know the real prevalence of CKD in this specific population. It must be reiterated here that the CKD-EPI equation with the African American patient correction factor has been developed and validated in cohorts including a vast majority of patients with CKD. We thus think that the current data are not suitable to determine the real performance of such an equation in African American patients who do not have CKD.

The authors also wrote, "Other equations were not considered because they are not more accurate than the CKD-EPI equation in external validation data sets and are not recommended by current guidelines." This is not fair. First, the authors should acknowledge that they were involved in establishing the guidelines. ${ }^{3}$ Second, the performances of other equations in determining GFR in African American people are simply missing, as these equations have been developed without consideration of African American people, and so without correction. At least the African American correction coefficient of the CKD-EPI equation could be tested with other equations. ${ }^{4}$ Third, good performance of other equations and the strategy of using no ethnic factor has been shown in African non-American populations. ${ }^{5}$ More fundamentally, correction by ethnic factors at the GFR level is misleading, as it suggests that GFR is different between patients of different races/ethnicities. Estimating GFR equations should correct for differences in serum creatinine generation, not for differences in GFR, as it has been shown that GFR is not different between white and black individuals. ${ }^{2,5}$ The full age spectrum equation (and its future development) is correcting for differences in creatinine generation using the median or mean serum creatinine of healthy populations (the $Q$ factor) as a scaling factor for serum creatinine in its formula. Therefore, the ethnic or population effect is integrated in this $Q$ value, at the creatinine level, not at the GFR level. ${ }^{5}$ Further studies on the performance of estimating GFR in African American patients and study participants seem to be required.

\section{Conflict of Interest Disclosures}

Dr Delanaye reported personal fees from Ark Biosciences, Immunodiagnostic Systems, AstraZeneca, Sanofi, Bayer, Amgen, Menarini Group, and Siemens HealthCare. No other disclosures were reported.

1. Levey AS, Tighiouart $\mathrm{H}$, Titan SM, Inker LA. Estimation of glomerular filtration rate with vs without including patient race. JAMA Intern Med. 2020;180(5): 793-795. doi:10.1001/jamainternmed.2020.0045 
2. Delanaye P, Mariat C, Maillard N, Krzesinski JM, Cavalier E. Are the creatinine-based equations accurate to estimate glomerular filtration rate in African American populations? Clin J Am Soc Nephrol. 2011;6(4):906-912. doi:10.2215/CJN.10931210

3. Levey AS, de Jong PE, Coresh J, et al. The definition, classification, and prognosis of chronic kidney disease: a KDIGO Controversies Conference report. Kidney Int. 2011;80(1):17-28. doi:10.1038/ki.2010.483

4. Erratum regarding "Comparing newer GFR estimating equations using creatinine and cystatin $\mathrm{C}$ to the CKD-EPI equation in adults" (Am J Kidney Dis. 2017;70[4]:587-589). Am J Kidney Dis. 2019;73(6):897. doi:10.1053/j.ajkd.2019.02.004

5. Bukabau JB, Yayo E, Gnionsahé A, et al. Performance of creatinine- or cystatin C-based equations to estimate glomerular filtration rate in sub-Saharan African populations. Kidney Int. 2019;95(5):1181-1189. doi:10.1016/j.kint.2018.11.045 